\title{
Circumstantial Evidence for Rotating Mass Matrix from Fermion Mass and Mixing Data
}

\author{
José BORDES \\ jose.m.bordes@uv.es \\ Departament Fisica Teorica, Universitat de Valencia, \\ calle Dr. Moliner 50, E-46100 Burjassot (Valencia), Spain \\ CHAN Hong-Mo \\ chanhm@v2.rl.ac.uk \\ Rutherford Appleton Laboratory, \\ Chilton, Didcot, Oxon, OX11 0QX, United Kingdom \\ and \\ NAPL, Department of Physics, \\ University of Oxford, Oxford, United Kingdom \\ TSOU Sheung Tsun \\ tsou@ maths.ox.ac.uk \\ Mathematical Institute, University of Oxford, \\ 24-29 St. Giles', Oxford, OX1 3LB, United Kingdom
}

\begin{abstract}
It is shown that existing data on the mixing between up and down fermion states and on the hierarchical mass ratios between fermion generations, as far as can be so analysed at present, are all consistent with the two phenomena being both consequences of a mass matrix rotating in generation space with changing energy scale. As a result, the rotating mass matrix can be traced over some 14 orders of magnitude in energy from the mass scale of the $t$-quark at $175 \mathrm{GeV}$ to below that of the atmospheric neutrino at $0.05 \mathrm{eV}$.
\end{abstract}




\section{Introduction}

Along with the mystery of why there should be in nature 3, and apparently only 3, generations of fermions, the fact that their masses should be hierarchical and that they should mix, as embodied for quarks in the CKM matrix [1] and exhibited for leptons in neutrino oscillations, has remained one of the great puzzles of particle physics. In the context of what we call the Dualized Standard Model (DSM) [2] which is an explicit attempt to solve this generation puzzle, we have suggested that the mass hierarchy and the mixing phenomenon can both result from a mass matrix which changes its orientation in generation space (rotates) with changing energy scale and have obtained rather good agreement with experiment based on this hypothesis. This previous treatment, however, does not fully cover the following two aspects. Firstly it depends on the details of the DSM mechanism driving the rotation, which details may not be strictly necessary for deriving the said result, and secondly, it does not clearly reveal the degree of significance of the claimed agreement with experiment nor the amount of direct empirical support, if any, for mass matrix rotation. For this reason, our purpose in this paper is to leave aside for the moment the driving mechanism (whether DSM or otherwise) but put instead the rotating mass matrix directly to the test by going straight to the experimental data and seek evidence there for the rotation hypothesis.

We shall show that by inputting all the available mass and mixing data on both quarks and leptons, assuming only that these all arise from mass matrix rotation in the manner to be explained below, one can trace the implied rotation over a scale range of some 14 orders of magnitude. The result is seen to be fully consistent with the fermion states all lying on a single smooth rotation curve linking the $t$ quark at $175 \mathrm{GeV}$ through all the intermediate fermion states down to the second heaviest neutrino $\nu_{2}$ at less than $10^{-2} \mathrm{eV}$. This constitutes positive evidence for mass matrix rotation, although as yet only circumstantial, which is independent of the theoretical mechanism responsible for driving it, whether DSM or otherwise. It will be seen, nevertheless, that the shape of the empirical rotation curve so traced is indicative of having the two rotational fixed points at infinite and zero energy scales predicted by and, as far as we know, special to the DSM scheme.

We begin by re-examining the reasoning behind the ansatz that both mass hierarchy and mixing can arise from a rotating mass matrix, for although it has been stated here and there and used already in several earlier papers, we cannot assume that it is of general knowledge. The first point to note is that 
once the mass matrix rotates with changing scale, then even such familiar concepts as particle masses, state vectors and mixing of flavour states, will have to be revised or refined, as we shall now explain.

Let us start with a fermion mass matrix traditionally defined by a term in the action of the form:

$$
\bar{\psi}_{L}^{0} m \psi_{R}^{0}+\text { h.c., }
$$

where $\psi_{L}^{0}$ and $\psi_{R}^{0}$ represent respectively the left- and right-handed fermion field, each being a vector in 3-dimensional flavour space, here given in the weak gauge basis, and $m$ is a $3 \times 3$ (complex) matrix. The matrix $m$ can always be diagonalized thus:

$$
U_{L}^{\dagger} m U_{R}=\operatorname{diag}\left\{m_{1}, m_{2}, m_{3}\right\}
$$

with $U_{L}, U_{R}$ unitary and $m_{i}$ real. Thus in terms of the fields:

$$
\psi_{L}=U_{L}^{\dagger} \psi_{L}^{0} ; \quad \psi_{R}=U_{R}^{\dagger} \psi_{R}^{0},
$$

the term (ㄱ) in the action takes on the diagonal form:

$$
\bar{\psi}_{L} \operatorname{diag}\left\{m_{1}, m_{2}, m_{3}\right\} \psi_{R} .
$$

When the mass matrix $m$ is constant in orientation with respect to scale change, i.e. in the language of this paper, when the mass matrix does not rotate, which is the simple case usually considered, then the particle masses of the 3 flavour states are just given by the diagonal values $m_{i}$. The above apply to both up and down quarks in the case of quarks, and to both charged leptons and neutrinos in the case of leptons. Hence, from the mass matrix, one obtains for the up and down states each a diagonalizing matrix $U_{L}$ which we can denote respectively as $U_{L}$ and $U_{L}^{\prime}$. Again, in the simple case when the mass matrices do not rotate, then the mixing matrix between up and down states (i.e CKM [1] for quarks and MNS [3] for leptons) is given just as [4]:

$$
V=U_{L} U_{L}^{\prime \dagger} .
$$

For the discussion in this paper, it is more convenient to work with an equivalent form of the mass matrix adopted by Weinberg in [5]. Since the right-handed fermion fields are flavour singlets, they can be arbitrarily relabelled without changing any of the physics. (Witness the fact that the mixing matrices between up and down states depend only on $U_{L}$ not on $U_{R}$.) Hence, 
by an appropriate relabelling of right-handed fields, explicitly by defining new right-handed fields:

$$
\psi_{R}^{\prime 0}=U_{L} U_{R}^{\dagger} \psi_{R}^{0},
$$

one obtains (11) in a form in which the the mass matrix becomes Hermitian:

$$
\bar{\psi} m_{W} \frac{1}{2}\left(1+\gamma_{5}\right) \psi+\bar{\psi} m_{W} \frac{1}{2}\left(1-\gamma_{5}\right) \psi=\bar{\psi} m_{W} \psi
$$

with

$$
m_{W}=m U_{R} U_{L}^{\dagger}
$$

This is convenient because in the simple case when the mass matrix does not rotate, the particle masses are now just the real eigenvalues of the Hermitian matrix $m_{W}$, as can readily be checked with (2). Furthermore, the mixing matrix between up and down states become just

$$
V_{i j}=\left\langle\mathbf{v}_{i} \mid \mathbf{v}_{j}^{\prime}\right\rangle
$$

with $\left|\mathbf{v}_{i}\right\rangle$ being the eigenvector of $m_{W}$ corresponding to the eigenvalue $m_{i}$, and the prime denoting down-type fermions. The scalar product $\left\langle\mathbf{v}_{i} \mid \mathbf{v}_{j}^{\prime}\right\rangle$ is of course an invariant independent of the frame in which these vectors are expressed. Thus, in terms of this Weinberg form of the mass matrix $m_{W}$ (which in this paper will be used exclusively and from which the subscript $W$ for convenience will henceforth be omitted), the mass values and state vectors of flavour states, as well as the mixing matrix between up and down fermions, are all easily defined in the case of no rotation in the mass matrix.

Consider now what happens in the case when the mass matrix rotates with changing scale as examined in this paper. Both its eigenvalues and their corresponding eigenvectors now change with the scale so that the previous definition of these as respectively the masses and state vectors of flavour states is no longer sufficiently precise, for it will have to be specified at which scale(s) the eigenvalues and eigenvectors are to be evaluated.

In the simple case of a single generation, i.e. when the the mass matrix is just a number, one is used to defining the particle mass as the running mass taken at the scale equal to the mass value itself, i.e. at that $\mu$ when $\mu=m(\mu)$. One might be tempted therefore to suggest for the multi-generation case that one defines the mass $m_{i}$ and the state vector $\mathbf{v}_{i}$ of the state $i$, as respectively just the $i$ th eigenvalue and eigenvector of the matrix $m$ taken at the scale $\mu_{i}=m_{i}\left(\mu_{i}\right)$, with $m_{i}(\mu)$ being the scale-dependent $i$ th eigenvalue of the matrix $m$. However, such a definition will not do, because it would mean that 
the state vectors for the different generations $i$ will be defined as eigenvectors of the matrix $m$ at different scales. Although the eigenvectors $i$ for different eigenvalues $i$ are orthogonal, $m$ being Hermition, when taken all at the same scale, they need not be mutually orthogonal when taken each at a different scale. But the state vectors for different flavour states ought to be orthogonal to one another if they are to be independent quantum states. Otherwise, it would mean physically that the flavour states (such as the charged leptons $e, \mu$ and $\tau$, for example) would have nonzero components in each other and be thus freely convertible into one another, or that the mixing matrices (CKM for quarks and MNS for leptons) would no longer be unitary, which would of course be unphysical.

How then should the mass values and state vectors of flavour states be defined in the scenario when the mass matrix rotates? To see how this question may be resolved, let us examine it anew with first the $U$-type quarks as example. The $3 \times 3$ mass matrix $m$ has 3 eigenvalues with the highest value $m_{1}$ corresponding to the eigenvector $\mathbf{v}_{1}$, both depending on scale $\mu$. Starting from a high scale and proceeding lower, one reaches at some stage $\mu_{1}=m_{1}\left(\mu_{1}\right)$, i.e. when the scale equals the highest eigenvalue $m_{1}$. One can then naturally define this value $m_{1}\left(\mu_{1}\right)$ as the $t$ quark mass $m_{t}$ and the corresponding eigenvector $\mathbf{v}_{1}\left(\mu_{1}\right)$ as the $t$ state vector $\mathbf{v}_{t}$. Next, how should one define the mass $m_{c}$ and the state vector $\mathbf{v}_{c}$ ? We have already seen above that they cannot be defined as respectively the second highest eigenvalue $m_{2}$ of the $3 \times 3$ mass matrix $m$ and its corresponding eigenvector at the scale $\mu_{2}=m_{2}\left(\mu_{2}\right)$, because this vector is in general not orthogonal to the state vector $\mathbf{v}_{t}$ which the state vector $\mathbf{v}_{c}$ ought to be. It is not difficult, however, to see what is amiss. At scales below the $t$ mass, i.e. when $\mu<m_{t}, t$ would no longer exist as a physical state, so that what functions there as the fermion mass matrix is not the $3 \times 3$ matrix $m$ but only the $2 \times 2$ submatrix, say $\hat{m}$, of $m$ in the subspace orthogonal to $\mathbf{v}_{t}$. Hence, for consistency, one should define $m_{c}$ as the highest eigenvalue $\hat{m}_{2}$ of the matrix $\hat{m}$ and the state vector $\mathbf{v}_{c}$ as the corresponding eigenvector, both at the scale $\hat{\mu}_{2}=\hat{m}_{2}\left(\hat{\mu}_{2}\right)$. The state vector of $c$ so obtained is of course automatically orthogonal to $\mathbf{v}_{t}$ as it should be. Repeating the argument, one defines further the mass $m_{u}$ and state vector $\mathbf{v}_{u}$ respectively as the "eigenvalue" and "eigenvector" of $\hat{\hat{m}}$ at the scale $\hat{\hat{\mu}}_{3}=\hat{\hat{m}}_{3}\left(\hat{\hat{\mu}}_{3}\right)$, with $\hat{\hat{m}}$ being the $1 \times 1$ submatrix of $m$ in the subspace orthogonal to both $\mathbf{v}_{t}$ and $\mathbf{v}_{c}$. Proceeding in this way, all masses and state vectors are defined at their own proper mass scale and the state vectors are mutually orthogonal as they should be. Besides, though stated above only for 3, the definition can be extended to any number of fermion generations, 
should there be physical incentive for doing so.

We note that a refined definition of masses and state vectors for flavour states is a question which has to be adressed in principle when the mass matrix rotates with changing scale, whatever the speed of the rotation. Hence, it ought to figure even in the conventional formulation of the Standard Model where the mass matrix is bound to rotate when there is nontrivial mixing between up and down states [6, 7], although the rotation there is rather slow and its effects are for most practical applications negligible. The above solution to the problem, though first made in the context of the DSM scheme [7], is seen actually to apply to any rotating mass matrix, and it is, as far as we know, the only solution to the question yet given in the literature. So this is the definition we shall employ in what follows to analyse existing mass and mixing data in terms of a rotating mass matrix. The success or otherwise of the analysis could thus itself be regarded as a check on the validity of the above solution.

Having now made clear our general procedure for defining masses and state vectors, let us return to the problem at hand, namely that of testing empirically the hypothesis that both mass hierarchy and mixing arise as consequences of the rotation of the mass matrix. First, by 'mixing arising from rotation', we mean that the mass matrices of up and down fermions taken at the same scale are aligned in orientation at all scales, only differing by a normalization factor, and it is the rotation alone which is giving rise to the mixing. Secondly, by 'mass mass hierarchy arising from rotation' as well, we mean that the mass matrix has at all scales only one massive eigenstate, with the masses of the lower generations appearing only by virtue of the rotation via what we called the "leakage" mechanism to be explained below. We have thus for both up and down fermions a mass matrix of the form:

$$
m=m_{T}|\mathbf{r}\rangle\langle\mathbf{r}|
$$

where $m_{T}$ is the single nonvanishing eigenvalue of $m$ and $|\mathbf{r}\rangle$ its corresponding (normalized) eigenvector. Or explicitly for 3 generations, we have:

$$
m=m_{T}\left(\begin{array}{l}
\xi \\
\eta \\
\zeta
\end{array}\right)(\xi, \eta, \zeta)
$$

where only $m_{T}$ depends on the fermion species. Thus the whole content of the rotating mass matrix is now encapsulated in $\mathbf{r}=(\xi, \eta, \zeta)$, a vector rotating in 3-dimensional generation space with changing scale $\mu$. 
That such a simple form of the mass matrix can nevertheless give rise to nontrivial mixing and mass hierarchy is most easily seen in a simplified situation when there are only 2 generations instead of 3. When applied to this simple case, the procedure detailed above for defining masses and state vectors of flavour states gives the state vector $\mathbf{v}_{t}$ of $t$ as the single massive eigenstate $\mathbf{r}$ of the $U$-quark mass matrix at the scale $\mu=m_{t}$, and the vector $\mathbf{v}_{c}$ as a vector orthogonal to $\mathbf{v}_{t}$, as depicted in Figure 11. Thus $\mathbf{v}_{c}$ has by (10) a zero eigenvalue for $m$ at the scale $\mu=m_{t}$. But according to our previous conclusion, this should not be interpreted to mean that $c$ has a zero mass. The $c$ mass $m_{c}$ is given instead as the eigenvalue of $\hat{m}$ at scale $\mu=m_{c}$, which in this simplified 2 generation case is just the expectation value of $m$ in the state $\mathbf{v}_{c}$. At the scale $\mu=m_{c}$, however, the vector $\mathbf{r}$ is already rotated to a direction different from that of $\mathbf{v}_{t}$, as illustrated in Figure 1, and has acquired a component equal to $\sin \theta_{t c}$ in the direction of $\mathbf{v}_{c}$, with $\theta_{t c}$ being the rotation angle between the scales $\mu=m_{t}$ and $\mu=m_{c}$. Hence, according to the above definition, one obtains "by leakage" a nonzero mass for $c$ given by the expectation value of (10) with respect to $\mathbf{v}_{c}$, namely:

$$
m_{c}=m_{t} \sin ^{2} \theta_{t c}
$$

Similarly, although the mass matrices of the $U$ and $D$ quarks according to (10) are aligned in orientation at all scales, one sees from Figure 2 that by virtue of the rotation of the vector $\mathbf{r}$ from the scale $\mu=m_{t}$ to the scale $\mu=m_{b}$ where the state vectors $\mathbf{v}_{t}$ and $\mathbf{v}_{b}$ are respectively defined, the two state vectors will not point in the same direction. One has thus from (9) simply by virtue of the rotation a nonzero mixing between the $t$ and $b$ states with the CKM matrix element:

$$
V_{t b}=\mathbf{v}_{t} \cdot \mathbf{v}_{b}=\cos \theta_{t b} \neq 1
$$

where $\theta_{t b}$ is the rotation angle between the two scales.

One sees already from these examples in the simplified scenario of only 2 generations that both lower generation masses and nontrivial mixing will automatically be obtained from a rotating mass matrix even if one starts with neither. One sees also that the same conclusion can be drawn in the 3 generation case so long as the masses and state vectors of flavour states are defined for a rotating mass matrix as detailed above. The remaining question to ask is then whether the actual masses and mixing of fermion states as observed in experiment can actually be explained in this simple way from a rotating mass matrix, and this is the question we set out to 


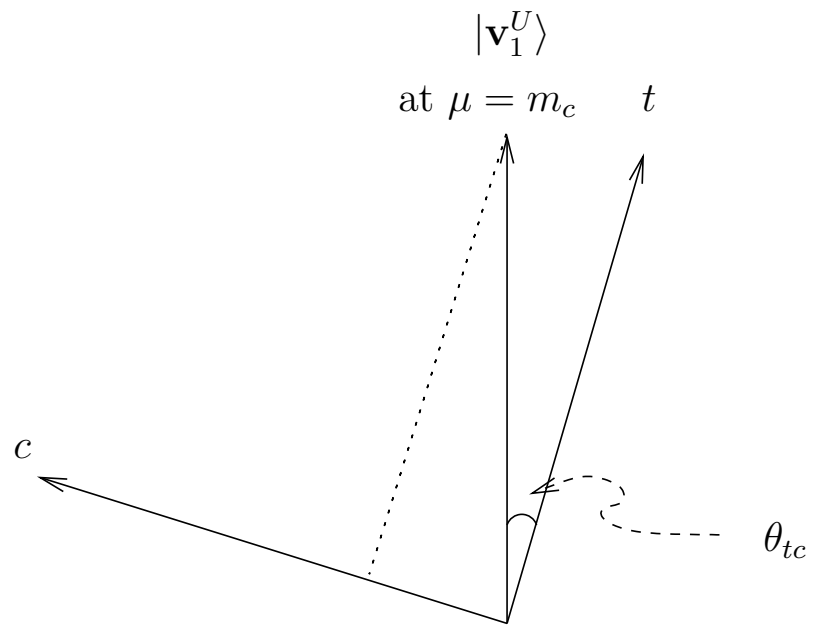

Figure 1: Masses for lower generation fermions from a rotating mass matrix via the "leakage" mechanism.

answer in this paper. This question can be adressed empirically since, as we shall show, many of the relevant quantities have already been measured and need only to be arranged and interpreted appropriately for the present purpose according to the definitions detailed above.

\section{Analysis in the planar approximation}

Let us first perform the analysis in the simplified situation with only the 2 heaviest generations in each fermion species, which scenario makes the analysis much more transparent since the problem then becomes planar and there is only one real rotation angle to consider [1, 4]. This simplification will be shown later to approximate already rather well the actual 3-generation situation in the scale region above roughly the $\mu$ lepton mass. We have then the pictures shown in Figures 11 and 2 for obtaining respectively the lower generation masses and mixing matrix elements.

Consider first mixing matrix elements. Suppose from the scale of the $t$ mass to that of the $b$ mass, the mass matrix has rotated by an angle $\theta_{t b}$ (Figure 21). As explained above, one easily obtains then the CKM elements as: $V_{t b}=\cos \theta_{t b}$ and $\left|V_{t s}\right|=\left|V_{c b}\right|=\sin \theta_{t b}$. From the measured values of these elements given in the latest databook [8], namely:

$$
\left|V_{t b}\right|=0.9990-0.9993, \quad\left|V_{t s}\right|=0.035-0.043, \quad\left|V_{c b}\right|=0.037-0.043,
$$




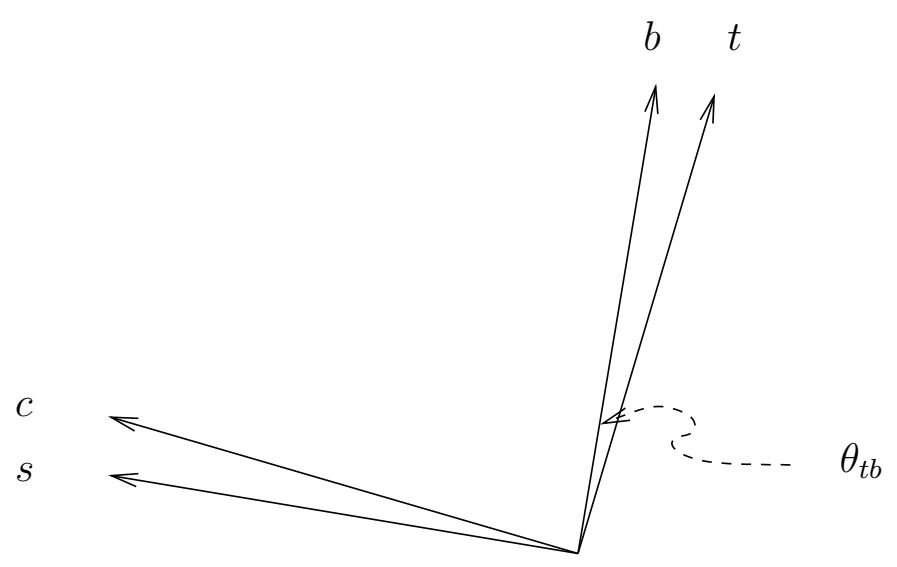

Figure 2: Mixing between up and down fermions from a rotating mass matrix.

one gets thus from each an estimate of the rotation angle, respectively:

$$
\theta_{t b}=0.0374-0.0447,0.0350-0.0430,0.0370-0.0430,
$$

the values obtained being fully consistent with one another. (One notes that from the same Figure 2, one could deduce in principle also $V_{c s}=\cos \theta_{t b}$, but this will be seen, in contrast to the 3 other mixing elements already considered, to be a poor approximation receiving large nonplanar corrections when all 3 generations are taken into account.)

Consider next the second generation masses obtained by the leakage mechanism. Suppose from the scale of the $t$ mass to that of the $c$ mass, the mass matrix has rotated by an angle $\theta_{t c}$. Using (12) and the measured values of $m_{t}$ and $m_{c}$ given in [8], namely:

$$
m_{t}=174.3 \pm 5.1 \mathrm{GeV}, \quad m_{c}=1.15-1.35 \mathrm{GeV} \text {, }
$$

one obtains the estimate:

$$
\theta_{t c}=0.0801-0.0894
$$

Similarly, from the measured values from [8]:

$$
m_{b}=4.0-4.4 \mathrm{GeV}, \quad m_{s}=75-170 \mathrm{MeV},
$$

one obtains the estimate:

$$
\theta_{b s}=0.1309-0.2076
$$


the error being so large because of the intrinsic uncertainty in defining the $s$ quark mass, while from the measured values from [8]:

$$
m_{\tau}=1.777 \mathrm{GeV}, \quad m_{\mu}=105.66 \mathrm{MeV},
$$

one obtains the estimate:

$$
\theta_{\tau \mu}=0.2463
$$

Assume now that the mass matrices of the $U$ and $D$ quarks as well as the charged leptons are all aligned at the same scale as proposed above, and plot the values of the rotation angles obtained before, all starting from the direction of the $t$ quark state. One obtains then the Figure 3 where, rotation angles being simply additive in the planar approximation, we have taken $\theta_{t s}=\theta_{t b}+\theta_{b s}$, and $\theta_{t \mu}=\theta_{t \tau}+\theta_{\tau \mu}$, with $\theta_{t b}$ taken from (15) and $\theta_{t \tau}$ (indicated by a cross in Figure 3) estimated by interpolation between the values of $\theta_{t b}$ and $\theta_{t c}$ given above. One sees first that the information gathered so far indeed appear consistent with the data points lying on a single smooth rotation curve as can be explained by a mass matrix rotating smoothly as the scale changes, which we regard as already a nontrivial support for the rotation hypothesis independent of any other theoretical consideration. In addition, one notes that the data by themselves are already indicative of the rotation angle approaching an asymptotic value thus suggesting a rotational fixed point at infinite scale as predicted by DSM. Indeed the data points all sit surprisingly well on the rotation curve obtained a few years earlier [9] in our DSM calculation. Making a best fit to the data by MINUIT produces an excellent fit:

$$
\theta=\exp (-2.267-0.509 \ln \mu)-0.0075
$$

for $\mu$ in $\mathrm{GeV}$, with a $\chi^{2}$ of 0.21 per degree of freedom, which is hardly distinguishable from the DSM curve, as can be seen in the same figure.

The above analysis was performed under the simplifying assumption of there being only 2 generations of fermion states but one can show that for the quantities so far considered it is already a good approximation to the actual 3-generation situation. When all 3 generations are taken into account, the mixing matrix can be parametrized as:

$$
\left(\begin{array}{ccc}
V_{t b} & V_{t s} & V_{t d} \\
V_{c b} & V_{c s} & V_{c d} \\
V_{u b} & V_{u s} & V_{u d}
\end{array}\right)=\left(\begin{array}{ccc}
c_{1} & -s_{1} c_{3} & -s_{1} s_{3} \\
s_{1} c_{2} & c_{1} c_{2} c_{3}-s_{2} s_{3} e^{i \delta} & c_{1} c_{2} s_{3}+s_{2} c_{3} e^{i \delta} \\
s_{1} s_{2} & c_{1} s_{2} c_{3}+c_{2} s_{3} e^{i \delta} & c_{1} s_{2} s_{3}-c_{2} c_{3} e^{i \delta}
\end{array}\right),
$$




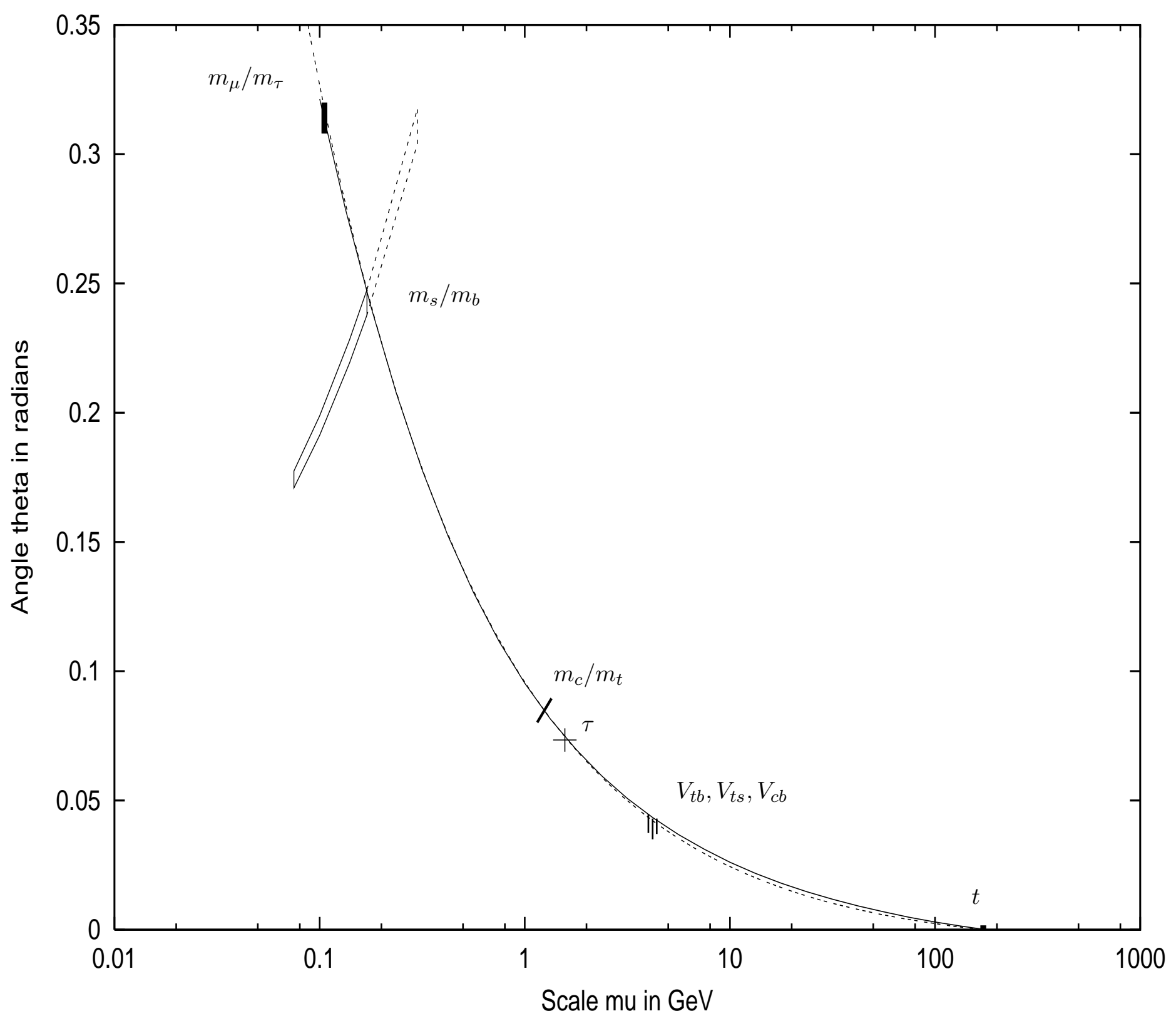

Figure 3: The rotation angle changing with scale as extracted from data on mass ratios and mixing angles and compared with the best fit to the data (dashed curve) and the earlier calculation by DSM (full curve) [9], in the planar approximation. 
which is the original Kobayashi-Maskawa parametrization [1], only with the ordering of fermion states reversed. One sees then that if we continue to denote as before $V_{t b}$ as $\cos \theta_{t b}$, the elements $V_{t s}$ and $V_{c b}$ are no longer just given by $\sin \theta_{t b}$ but by respectively $\sin \theta_{t b} \cos \theta_{3}$ and $\sin \theta_{t b} \cos \theta_{2}$. The angles $\theta_{2}$ and $\theta_{3}$, however, are small as can be estimated from the empirical values given in [8] for the corner elements of the CKM matrix in comparison to the values of $V_{t s}$ and $V_{c b}$ quoted above, giving:

$$
\begin{aligned}
& \left|V_{t d}\right|=0.004-0.014 \longrightarrow\left|\tan \theta_{3}\right|=0.093-0.400, \\
& \left|V_{u b}\right|=0.002-0.005 \longrightarrow\left|\tan \theta_{2}\right|=0.047-0.135,
\end{aligned}
$$

from which one gets:

$$
\cos \theta_{2}=0.999-0.991 ; \quad \cos \theta_{3}=0.996-0.928 \text {. }
$$

Hence, one concludes that in the 2 generation planar approximation of Figures 2 and 3 where one puts $V_{t s}=V_{c b}=\sin \theta_{t b}$, one has made an error of at most a few percent which is seen hardly to affect the plot or any of the previous remarks we made. A similar error has been made in Figure 3 as regards the lower generation masses obtained from the "leakage" mechanism. The estimate (19) is for the angle rotated between the scales of $m_{b}$ and $m_{s}$ but in Figure 3 we have added this angle to the rotation angle from scale $m_{t}$ to scale $m_{b}$ to get the angle from scale $m_{t}$ to scale $m_{s}$. Such an addition is valid in the 2 generation approximation but has nonplanar corrections in the actual 3 generation situation. The error so incurred can be estimated as follows. The angle between the plane defined by the $t$ and $c$ vectors and the plane defined by $b$ and $s$ is given by the angle between their normals, namely the vectors for $u$ and $d$ respectively, which according to [8] takes the value:

$$
\left|V_{u d}\right|=\cos \theta_{u d}=0.9742-0.9757
$$

giving

$$
\theta_{u d}=0.2209-0.2276 .
$$

The nonplanar error incurred in the angle at scale $m_{s}$ plotted in Figure 3 is of order $1-\cos \theta_{u d}$ and is thus of order again a few percent, which is negligible given the large error already inherent in the definition of the $s$ quark mass. A similar error is presumably present in the angle plotted in Figure 3 at scale $m_{\mu}$. More details of this analysis can be found in a preliminary report [10].

However, though good for illustration purposes because of its simplicity, the above analysis in the planar approximation is incomplete in that it is 
restricted only to scales above the $\mu$ mass where nonplanar effects are negligible, and hence cannot account for all the available empirical information which includes the data from neutrino oscillations at very low scales where nonplanar effects can no longer be neglected. Furthermore, to test the rotation hypothesis exhaustively one has also to ensure that no hidden violation of the hypothesis exists in the off-planar direction. In any case, the planar approximation, though good for illustrative purposes, actually need not be made since, as detailed in (10) above, the whole content of the rotating mass matrix even when all 3 generations are taken into account is encapsulated just in the single 3 -dimensional vector $\mathbf{r}(\mu)$ depending on scale $\mu$. The only technical problem posed by a full analysis is thus how to extract this vector at various scales from the existing data on fermion mass ratios and mixing parameters. Once so extracted, the vector can be confronted with the rotation hypothesis and should be consistent with tracing out a continuous curve in 3-space if the hypothesis is correct. For the extraction of this vector, we now proceed as follows.

\section{Extracting $\mathbf{r}(\mu)$ from quark data}

The $U$-quarks $t, c, u$ are independent quantum states so that their state vectors should form an orthonormal triad in generation space, which we can choose without loss of generality as:

$$
\mathbf{v}_{t}=(1,0,0) ; \quad \mathbf{v}_{c}=(0,1,0) ; \quad \mathbf{v}_{u}=(0,0,1)
$$

The $D$-quark state vectors also form an orthonormal triad the orientation of which relative to the $U$-triad is given by the CKM matrix elements:

$$
\mathbf{v}_{b}=\left(V_{t b}, V_{c b}, V_{u b}\right) ; \quad \mathbf{v}_{s}=\left(V_{t s}, V_{c s}, V_{u s}\right) ; \quad \mathbf{v}_{d}=\left(V_{t d}, V_{c d}, V_{u d}\right) .
$$

Hence, if the complex elements of the CKM matrix are accurately known, the $D$-triad would also be determined. At present, however, only the absolute values of the CKM matrix elements are experimentally known to reasonable accuracy, leading thus to some ambiguities in the determination of the $D$ triad. In particular, one is forced to ignore for the moment in the CKM matrix the $\mathrm{CP}$-violating phase which is experimentally very poorly determined and treat the $D$-triad also as real vectors. Inserting then the experimental limits on the CKM matrix elements as read from [8] gives rather tight constraints on the directions of the $D$-triad with errors so small as to be mostly 
negligible for our analysis:

$$
\begin{aligned}
& \mathbf{v}_{b}=((0.9990-0.9993),(0.037-0.043),-(0.002-0.005)) \\
& \mathbf{v}_{s}=(-(0.035-0.043),(0.9734-0.9749),(0.219-0.226)) \\
& \mathbf{v}_{d}=((0.004-0.014),-(0.219-0.225),(0.9742-0.9757))
\end{aligned}
$$

The signs of the 3 components for $b$ can be chosen arbitrarily by choosing the physically irrelevant phases of the various quark state vectors and a particular choice has been made in (30) for convenience. The signs for the other 2 states are then determined by orthogonality. Actually, given the present errors on the CKM matrix elements, there is an alternative solution to that shown for the state vectors of $s$ and $d$, which however we can ignore for reasons to be explained below.

We notice that by the considerations in the preceding section, the state vector of $b$, this being the heaviest state in the $D$ sector, is just the rotating vector $\mathbf{r}(\mu)$ taken at the scale $\mu=m_{b}$, thus:

$$
\mathbf{r}\left(m_{b}\right)=\mathbf{v}_{b} .
$$

Together with:

$$
\mathbf{r}\left(m_{t}\right)=\mathbf{v}_{t}=(1,0,0)
$$

we have then two points on the trajectory for $\mathbf{r}(\mu)$ we wish to trace. One convenient way to present this, we find, is to write this rotating vector as $\mathbf{r}(\mu)=(\xi(\mu), \eta(\mu), \zeta(\mu))$ with $\xi(\mu)^{2}=1-\eta(\mu)^{2}-\zeta(\mu)^{2}$ and plot the constraints on $\eta(\mu)$ and $\zeta(\mu)$ on the $\eta \zeta$-plane. The result from (32) and (31) are then entered as the first 2 points from the left in the 3-D plot of Figure 4. This plot, which shows $\eta(\mu)$ and $\zeta(\mu)$ as functions of the energy scale $\mu$ can in principle incorporate all the information that we shall extract from data. However, it being often hard to read the information it contains, we shall supplement it by its 3 projections onto the 3 co-ordinate planes, namely onto the $\eta \zeta$-plane in Figure 5, the $\mu \eta$-plane in Figure 6, and the $\mu \zeta$-plane in Figure 0, which projections, as we shall see, will be useful later also for interpolation and extrapolation purposes.

Next, we turn to consider $\mathbf{r}(\mu)$ at the scale $\mu=m_{c}$. Since the mass of $m_{c}$, by our original hypothesis, comes about only through the "leakage" from $m_{t}$ as detailed above, it follows that the vector $\mathbf{r}\left(m_{c}\right)$ will have to be a linear combination: $\cos \theta_{t c} \mathbf{v}_{t}+\sin \theta_{t c} \mathbf{v}_{c}$, of the state vectors $\mathbf{v}_{t}$ and $\mathbf{v}_{c}$ of respectively $t$ and $c$ with $\sin \theta_{t c}$ given by (12), or explicitly:

$$
\mathbf{r}\left(m_{c}\right)=\sqrt{1-m_{c} / m_{t}} \mathbf{v}_{t}+\sqrt{m_{c} / m_{t}} \mathbf{v}_{c} .
$$




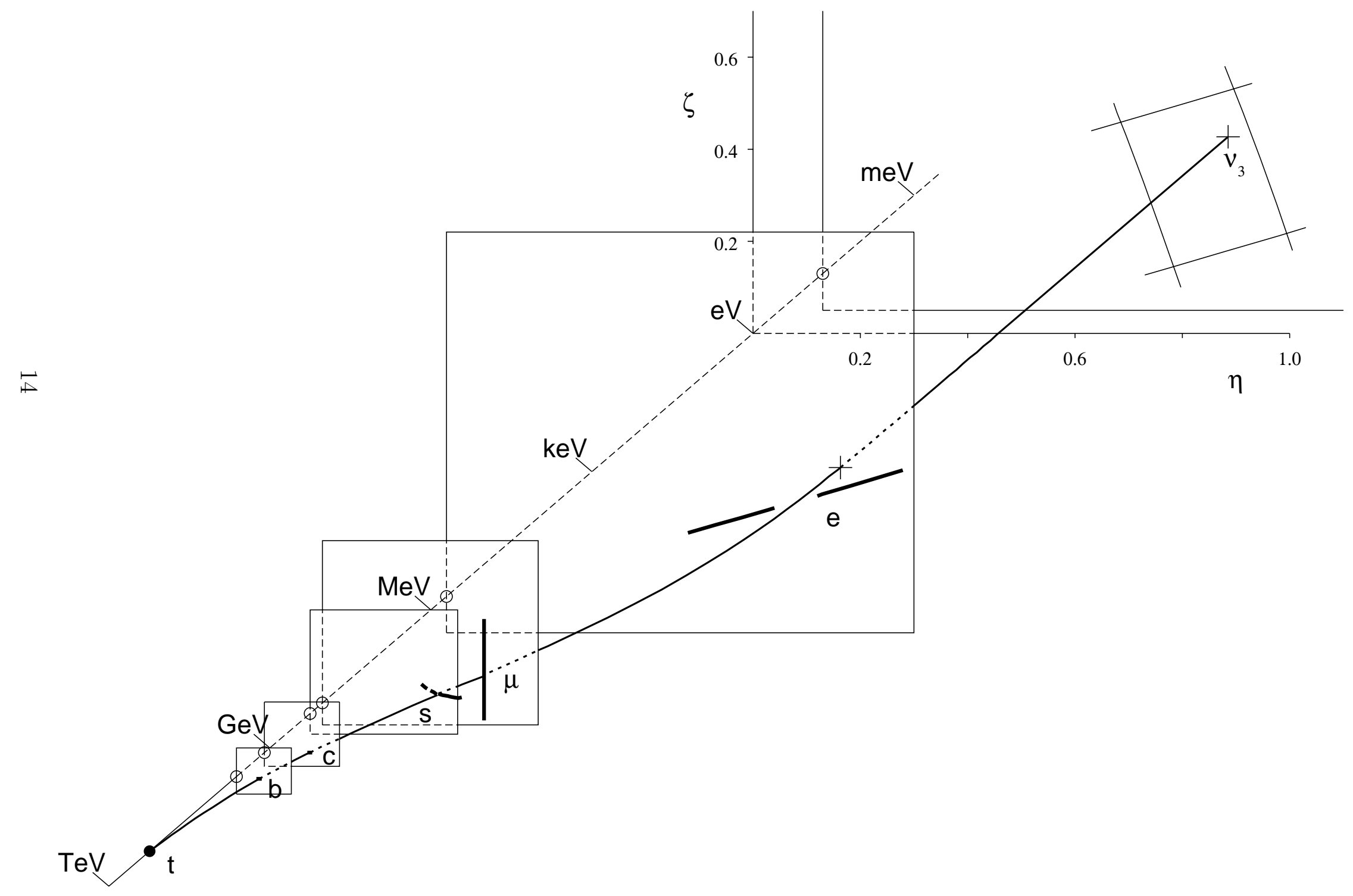


Figure 4: A plot of the rotating vector $\mathbf{r}(\mu)$ as extracted from existing data on fermion mass ratios and mixing parameters, where its second and third components, i.e. $\eta(\mu)$ and $\zeta(\mu)$, are plotted as functions of $\ln \mu, \mu$ being the energy scale. The experimentally allowed values at any one scale are represented as an allowed region on a plaquette, with the scale corresponding to a plaquette being given by the intersection, denoted by a small circle, of its left-most boundary with the $\mu$-axis. For example, the first small plaquette on the left of the figure corresponds to the scale $\mu=m_{b}$, on which plaquette the allowed region for $\mathbf{r}(\mu)=\mathbf{v}_{b}$ is very small because of the small experimental error on the CKM matrix elements $V_{t b}, V_{c b}$ and $V_{u b}$. The last plaquette on the right, on the other hand, corresponds to the scale $\mu=m_{\nu_{3}}$, on which plaquette the allowed region for $\mathbf{r}(\mu)$ is a rough rectangular area bounded by the data on $\nu$ oscillations from atmospheric neutrinos and from the Chooz experiment. The curve represents the result of a DSM one-loop calculation from an earlier paper [9] which is seen to pass through the allowed region on every plaquette except that for the electron $e$. For further explanation of details, please see text. 


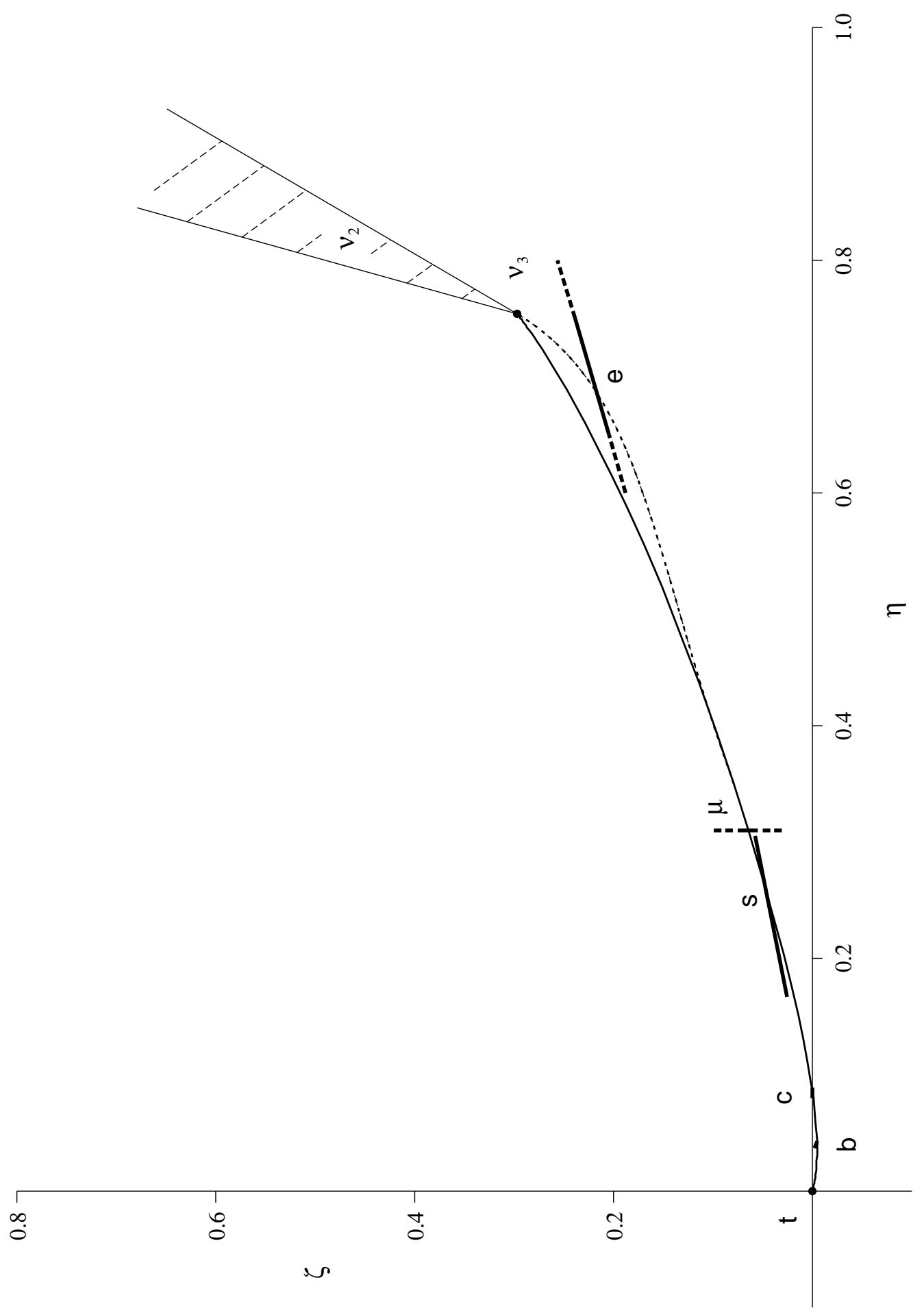

Figure 5: Projection of Figure 1 onto the $\eta \zeta$-plane. The full curve represents the DSM one-loop calculation of [9] and the dashed curve its suggested deformation at low scales to fit the data on $m_{e}$ and $U_{e 2}$. 


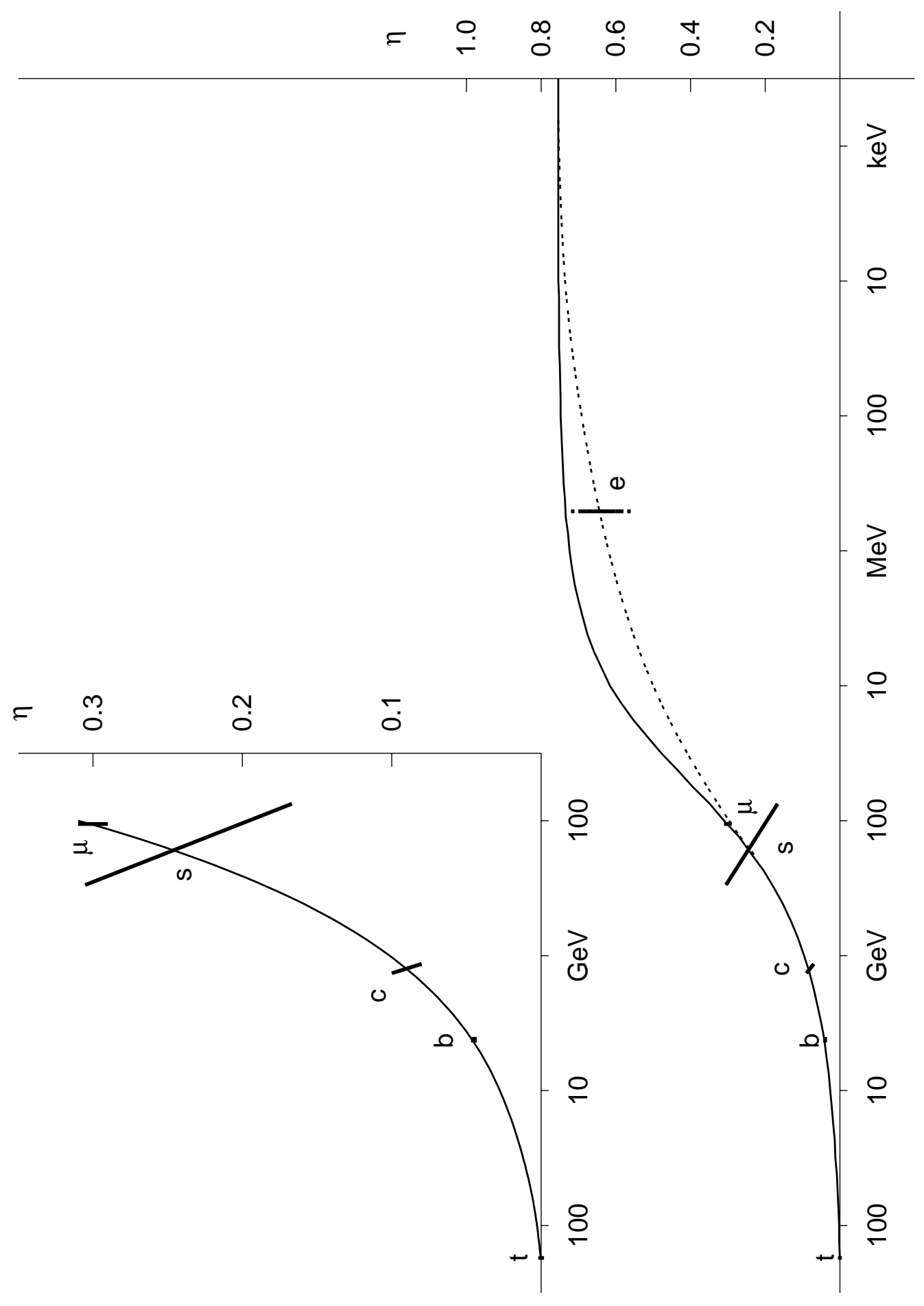

Figure 6: Projection of Figure 4 onto the $\mu \eta$-plane. The full curve represents the DSM one-loop calculation of [9] and the dashed curve its suggested deformation at low scales to fit the data on $m_{e}$ and $U_{e 2}$. 


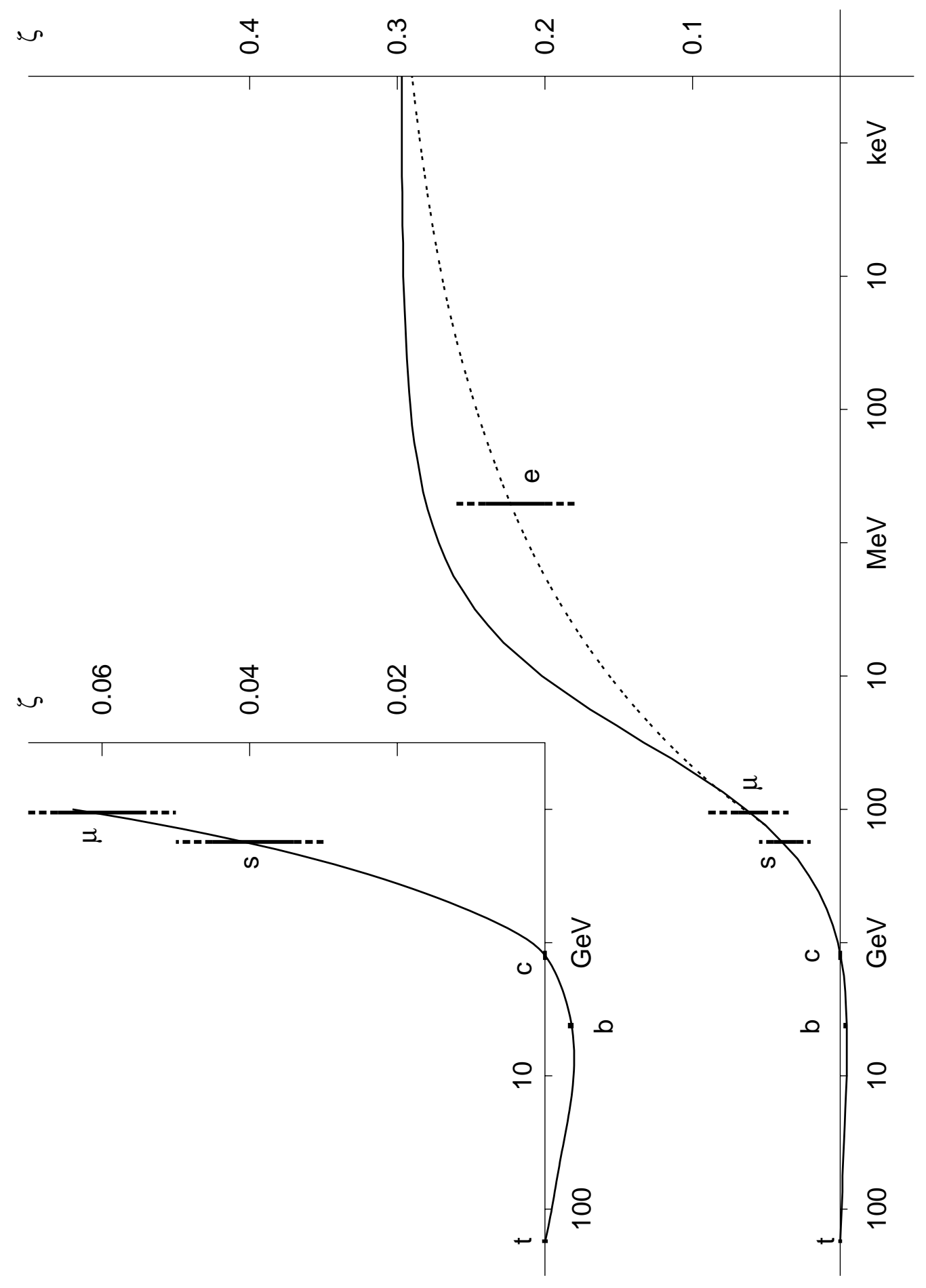

Figure 7: Projection of Figure 6 onto the $\mu \zeta$-plane. The full curve represents the DSM one-loop calculation of [9] and the dashed curve its suggested deformation at low scales to fit the data on $m_{e}$ and $U_{e 2}$. 
Inputting the quoted experimental limits on $m_{t}$ and $m_{c}$ gives us the allowed region for the third data point on the trajectory of $\mathbf{r}(\mu)$, as is shown on Figure . There is in fact of course another solution for $\mathbf{r}\left(m_{c}\right)$ in (33) with $\sin \theta_{t c}=-\sqrt{m_{c} / m_{t}}$, meaning that there is an additional disjoint branch of the allowed region in another quadrant of the $\eta \zeta$-plane to that displayed in the figure. The existence of this additional branch, however, does not affect the question of interest to us here, namely, whether the allowed region is consistent with data lying on a smooth rotation curve, so long as the first branch already does, and can therefore be ignored. Such additional branches of the allowed regions will in fact always occur for all other pieces of information on $\mathbf{r}(\mu)$ that we shall extract from data, since experiment so far gives only the absolute values of the relevant quantities but, for the same reason as above, these additional disjoint branches for our present purpose can almost always be ignored.

The next point in line is $\mathbf{r}(\mu)$ at $\mu=m_{s}$, which will of necessity be poorly determined because the $s$ mass is very poorly known. Nevertheless, whatever is taken for the mass of $s$ so long as it is given by the "leakage mechanism" from $b, \mathbf{r}\left(m_{s}\right)$ will have to be a linear combination of the state vector $\mathbf{v}_{b}$ of $b$ and the state vector $\mathbf{v}_{s}$ of $s$, so that by reasoning exactly as above for $c$, we have:

$$
\mathbf{r}\left(m_{s}\right)=\sqrt{1-m_{s} / m_{b}} \mathbf{v}_{b}+\sqrt{m_{s} / m_{b}} \mathbf{v}_{s} .
$$

The range of values for $m_{s}$ is given in [8] as 75-170 MeV, and in [11] as $100-300 \mathrm{MeV}$, depending on the scale at which the limits were determined respectively. The allowed region shown for $\mathbf{r}\left(m_{s}\right)$ in Figure 4 corresponds to the union of the above two ranges for $m_{s}$ values, which allowed region, one will notice, does not lie entirely on the plaquette corresponding to an $m_{s}$ value at $176 \mathrm{MeV}$ but protrudes to either side of it. This gives then a 4 th point on the trajectory for $\mathbf{r}(\mu)$. As noted before, given the present errors on the CKM mattrix elements, there is actually an alternative solution for $\mathbf{v}_{s}$ with a different sign for the third component to that given in (30), meaning an additional branch to the allowed region for $\mathbf{r}\left(m_{s}\right)$, but this can be ignored for the same reason as that given for $\mathbf{r}\left(m_{c}\right)$ above.

The above represents more or less the most that can be extracted from the data on quark masses and mixing (barring $\mathrm{CP}$ violation) about the rotating vector $\mathbf{r}(\mu)$ apart from some as yet rather uncertain information from the masses of the light quarks $u$ and $d$. The method we used for extracting the fermion masses from the "leakage mechanism" as contained in e.g. (12) was meant only for freely propagating particles and should not in principle be 
applied to quarks which are confined, except approximately to the heavier quarks which are generally regarded as quasi-free. For the light quarks $u$ and $d$ which are tightly confined, it is clearly not applicable. The question then arises in what way these light quark masses are to be defined. Experimentally, these masses are determined at some convenient but somewhat arbitrary scale such as 1 or $2 \mathrm{GeV}$, and it is not clear what these values should correspond to in the "leakage mechanism". One possibility is to consider these mass values as the "leakage" from the rotating vector $\mathbf{r}(\mu)$ taken at the chosen scale 1 or $2 \mathrm{GeV}$ into the vectors $\mathbf{v}_{u}$ and $\mathbf{v}_{d}$ thus:

$$
\left|\mathbf{v}_{u} . \mathbf{r}(\mu)\right|^{2} \stackrel{?}{=} m_{u} / m_{t} ; \quad\left|\mathbf{v}_{d} \cdot \mathbf{r}(\mu)\right|^{2} \stackrel{?}{=} m_{d} / m_{b}
$$

In that case one obtains values for both $m_{u}$ and $m_{d}$ of the right order of magnitude in the $\mathrm{MeV}$ region, but it is not certain whether this is of much significance. As matters stand, therefore, we can only leave open the question of the light quark masses.

\section{Extracting $\mathbf{r}(\mu)$ from lepton data}

To proceed further, one turns now to the leptons for which the preceding analysis for quarks can in principle be independently repeated, for as far as the premises of the rotating mass matrix as set up at the beginning of this paper is concerned, there is strictly nothing which needs connect the mass matrices of quarks and leptons. However, the DSM scheme suggests that the rotating matrices for quarks and leptons both lie on the same trajectory, the last being specified just by the vev's of the (dual colour) Higgs fields which are independent of the fermion type [12, 9], and this suggestion has been borne out by the 2-generation analysis reported above. It makes practical sense therefore to adopt the same position here, especially since it raises the stakes and makes the present analysis an even more stringent test for the rotation hypothesis. This means in particular that the state vector, say $\mathbf{v}_{\tau}$, of the $\tau$ lepton, this being the heaviest eigenstate of the lepton mass matrix, could be identified again with the vector $\mathbf{r}(\mu)$ taken at the scale $\mu=m_{\tau}$, the location of which can readily be determined by interpolating $\mathbf{r}(\mu)$ between $m_{b}$ and $m_{c}$, as in Figure 3 .

Having fixed $\mathbf{v}_{\tau}$, one can constrain the vector $\mathbf{r}(\mu)$ at $\mu=m_{\mu}$ by the condition:

$$
\left|\mathbf{r}\left(m_{\mu}\right) \cdot \mathbf{v}_{\mu}\right|^{2}=m_{\mu} / m_{\tau}
$$


with:

$$
\mathbf{v}_{\mu} \cdot \mathbf{v}_{\tau}=0
$$

or in other words:

$$
1-\left|\mathbf{r}\left(m_{\mu}\right) \cdot \mathbf{v}_{\tau}\right|^{2}=m_{\mu} / m_{\tau}
$$

This gives 4 solutions for $\eta\left(m_{\mu}\right), \zeta\left(m_{\mu}\right)$, corresponding to respectively the two signs of $\mathbf{r}\left(m_{\mu}\right) \cdot \mathbf{v}_{\tau}$ and the two signs of $\xi\left(m_{\mu}\right)= \pm \sqrt{1-\eta\left(m_{\mu}\right)^{2}-\zeta\left(m_{\mu}\right)^{2}}$. These 4 solution are, however, widely separated, so that only the solution with $\mathbf{r}\left(m_{\mu}\right) \cdot \mathbf{v}_{\tau}$ and $\xi\left(m_{\mu}\right)$ both positive is shown in Figure 1 which, by inputting the empirical values of $m_{\tau}=1777 \mathrm{MeV}$ and $m_{\mu}=105 \mathrm{MeV}$ taken from [8], gives as the allowed region for $\mathbf{r}\left(m_{\mu}\right)$ a narrow band on the $\mu$ plaquette approximately parallel to the $\zeta$-axis, the width of the band representing the error on $\mathbf{v}_{\tau}$ obtained from the above interpolation. For the rotation hypothesis to be valid, the trajectory for $\mathbf{r}(\mu)$ is required to pass through this band at $\mu=m_{\mu}$.

The above information on the vector $\mathbf{r}\left(m_{\mu}\right)$ determines also to a fair approximation the state vector $\mathbf{v}_{\mu}$, the latter being constrained by the "leakage" mechanism to lie on the plane containing $\mathbf{v}_{\tau}$ and $\mathbf{r}\left(m_{\mu}\right)$ and to be orthogonal to $\mathbf{v}_{\tau}$. That this is so can be seen as follows. As noted already, the vector $\mathbf{v}_{\tau}$ being near the vector $\mathbf{r}\left(m_{c}\right)$ and therefore lying very nearly on the $\xi \eta$-plane, the allowed band for $\mathbf{r}\left(m_{\mu}\right)$ defined by (38) is very nearly parallel to the $\zeta$-axis so that the second component of $\mathbf{r}\left(m_{\mu}\right)$, namely $\eta\left(m_{\mu}\right)$, is very well determined, as is depicted in Figure 6. By interpolating with a curve drawn through the four quite accurate points for respectively $t, b, c$ and $\mu$, one can then get a fair estimate for the value of $\eta\left(m_{s}\right)$. Hence from Figure 5, one can read off the corresponding value for the third component $\zeta\left(m_{s}\right)$ which on insertion into Figure 7 then allows for an extrapolation to the $\mu$ mass scale to give an estimate for the value of $\zeta\left(m_{\mu}\right)$, which though rough, being in any case small, is sufficient for our purpose. Having then obtained the vector $\mathbf{r}\left(m_{\mu}\right)$, the state vector of $\mu$, namely $\mathbf{v}_{\mu}$, is also determined by the conditions stated at the beginning of the paragraph. At the same time, of course, the state vector $\mathbf{v}_{e}$ of $e$ is also determined by orthogonality to both $\mathbf{v}_{\mu}$ and $\mathbf{v}_{\tau}$. The actual numerical values we so obtained for the charged leptonic triad which we shall use later for our analysis are as follows:

$$
\begin{aligned}
& \mathbf{v}_{\tau}=(0.9975,0.0700,-0.0015) \\
& \mathbf{v}_{\mu}=(-0.0654,0.9516,0.3003) \\
& \mathbf{v}_{e}=(0.0224,-0.2995,0.9538)
\end{aligned}
$$


This determination of the charged lepton triad on which the analysis in the remainder of this section depends is about the best that one can do for the moment but is obviously not as accurate as one could wish. Nevertheless, as we shall see, it still serves its purpose in allowing us to extract some interesting information on $\mathbf{r}(\mu)$ for the low $\mu$ region.

First, according to the "leakage mechanism", the mass of the electron is given by:

$$
\left|\mathbf{r}\left(m_{e}\right) \cdot \mathbf{v}_{e}\right|^{2}=m_{e} / m_{\tau}
$$

which, as for (36) and for the same reasons, gives 4 solutions, 2 of which corresponding to negative values for $\xi\left(m_{e}\right)$ lie outside Figure 1 , leaving two which are represented by respectively the line drawn on the $e$-plaquette and another line (not shown) nearly parallel to the first but 0.035 units lower (and hence almost coinciding with the one shown). Again, for the rotation hypothesis to be valid, the trajectory for $\mathbf{r}(\mu)$ has to pass through one of these 2 line at $\mu=m_{e}$. In obtaining these lines, we have of course input the well known value of $0.51 \mathrm{MeV}$ for the mass of the electron.

Secondly, the MNS [3] lepton mixing matrix elements $U_{\mu 3}$ and $U_{e 3}$, as studied in oscillation experiments on respectively atmospheric neutrinos 13, 14] and reactor neutrinos such as [15], are given by the inner products:

$$
U_{\mu 3}=\mathbf{v}_{\mu} \cdot \mathbf{v}_{3},
$$

and:

$$
U_{e 3}=\mathbf{v}_{e} \cdot \mathbf{v}_{3},
$$

with $\mathbf{v}_{3}=\mathbf{r}\left(m_{\nu_{3}}\right)$ being the state vector of the heaviest neutrino $\nu_{3}$. With the $\mu$ state vector as determined above in (39), one obtains by inputting the experimental range for $\left|U_{\mu 3}\right|^{2}$ of about $1 / 3$ to $2 / 3$ [13, 14 again four solutions for the allowed region, three of which lie outside Figure 4 , leaving one (corresponding to $U_{\mu 3}$ and $\xi\left(m_{\nu_{3}}\right)$ both positive) which is represented in the figure by the area bounded by the two near vertical lines on the $\nu_{3}$-plaquette. Similarly, with the $e$ state vector as determined in (39), one obtains by inputting the experimental bound $\left|U_{e 3}\right|^{2}<0.027$ [15], four solutions for the allowed region, but this time only two (corresponding to $\xi\left(m_{\nu_{3}}\right)$ negative) can be ignored being outside Figure $₫$, the other two being adjacent merge into one as represented in the figure by the area bounded by the two near horizontal lines on the $\nu_{3}$-plaquette. The consequent allowed region for the vector $\mathbf{r}\left(m_{\nu_{3}}\right)$ is thus represented by the roughly rectangular area shown, where we have put $m_{\nu_{3}}^{2} \sim 3 \times 10^{-3} \mathrm{eV}^{2}$ as preferred by [13, 14] and [16]. 
Finally, the mixing element $U_{e 2}$ as inferred from solar neutrino experiments is given as:

$$
U_{e 2}=\mathbf{v}_{e} \cdot \mathbf{v}_{2},
$$

where $\mathbf{v}_{2}$ is the state vector for the second heaviest neutrino $\nu_{2}$ which is by definition orthogonal to $\mathbf{v}_{3}$. Following thus the same procedure as in the preceding paragraph, one can determine the allowed region for the vector $\mathbf{v}_{2}$ by inputting the bounds on $\mathbf{v}_{3}$ as obtained above and the experimental bounds on $U_{e 2}$ [13, 17, 18]. However, to extract $\mathbf{r}\left(m_{\nu_{2}}\right)$ from this, one would need $m_{\nu_{2}}$ which is experimentally still largely unknown so that the above information on $\mathbf{v}_{2}$ cannot readily be presented in Figure . . But, as we shall see, there is another way of displaying this information.

\section{Discussion}

The allowed regions for the vector $\mathbf{r}(\mu)$ for various scales $\mu$ displayed in Figure 1 and its projections Figures 5 , 7 , represent all the information on $\mathbf{r}(\mu)$ that can be extracted at present from fermion mass and mixing parameters, apart from the $u, d$ masses and the solar neutrino angle $U_{e 2}$ already noted. This information was extracted on the assumption that both fermion mixing and lower generation masses arose solely as consequences of the rotation of the mass matrix, under which circumstances the rotation is encapsulated entirely in the rotating vector $\mathbf{r}(\mu)$, as was explained in equation (10) or (11). The definition of masses, state vectors, and mixing matrices, which for a rotating mass matrix is delicate, followed the prescription given in the introduction, which seems to us the natural one and is to our knowledge the only one available in the literature. Apart from these, no other theoretical input or assumption has been introduced. Hence if the rotation hypothesis set out above is correct, then the allowed regions should line up along some smooth 3-D curve from the heaviest $t$ to the lowest $\nu_{3}$. Indeed this is seen in the above-quoted figures to be the case. In the high energy region, say down to the $\mu$ lepton mass scale, where the allowed regions are mostly small, the alignment is seen to be quite accurate, not only in the projection of Figure 6 on to the $\mu \eta$-plane as already noted in Section 2, but also in the two other directions as seen in Figures 5 and 7 . Below the $\mu$ lepton mass scale, the allowed regions are larger and the constraints not too stringent but they are seen still to be thoroughly consistent with alignment on a smooth trajectory spanning some 13 orders of magnitude in energy. This looks to us nontrivial and lends direct empirical support to the rotation hypothesis 
which is entirely model-independent and free from extraneous theoretical bias. This is the main conclusion we set out to establish.

Next, we turn to consider the shape of the trajectory traced out by the data in Figure 1 , to understand which more theoretical input will of course be needed. We shall do so with reference to our DSM scheme where the whole rotation idea was first proposed and in which a perturbative method for calculating the rotating trajectory was suggested and carried out already to 1-loop order 12, 9]. First, we notice from Figures and that $\mathbf{r}(\mu)$ seems to be approaching asymptotic limits for both $\mu \rightarrow \infty$ and $\mu \rightarrow 0$, and thus indicative of rotation fixed points at these scale values. Now, these fixed points are predicted by the DSM scheme, and they occur there by virtue only of the inbuilt mechanism in the model for driving the rotation and are thus independent of the adjustable parameters of the model. The detailed shape of the trajectory, however, does depend on the parameters of the model, of which there are 3 , with 2 giving the initial direction of the trajectory and 1 other governing the rotation speed. The calculation done already a few years ago [9] with the 3 parameters in the model fitted to the mass ratios $m_{c} / m_{t}$, $m_{\mu} / m_{\tau}$ and to the Cabibbo angle is reproduced in Figures 4 7. Although this result has never been explicitly presented before, it can be inferred from e.g. Figure 3 of [9] and transformed to the present frame (28) from the frame used there through the vectors:

$$
\begin{aligned}
\mathbf{v}_{t} & =(0.9999,0.0117,0.0008) \\
\mathbf{v}_{c} & =(-0.0110,0.9148,0.4038) \\
\mathbf{v}_{u} & =(0.0040,-0.4038,0.9149)
\end{aligned}
$$

obtained from the previous calculation [9]. It is seen to agree very well with the newly extracted information down to the $\mu$ mass scale. In particular, the rotational fixed point predicted by the DSM at $\mu=\infty$ is seen to be fully consistent with the data. Below the $\mu$ mass the DSM curve calculated to 1-loop order begins to deviate from the regions allowed by experiment. For example, on the e-plaquette in Figure 1, the DSM curve if exact should hit the allowed line at $\mu=m_{e}$ but, as indicated by the little cross, it hits the plaquette instead at some distance from the allowed line. This deviation represents the difference in the mass of the electron as predicted by the old calculation 9] from its true value, i.e. $6 \mathrm{MeV}$ instead of $0.51 \mathrm{MeV}$. Such a deviation is of course expected, since at lower scales, the vector $\mathbf{r}(\mu)$ moves further and further from the high energy fixed point predicted by the scheme so that the 1-loop calculation for the trajectory will become less and less 
reliable. However, the 1-loop approximate trajectory from s. still hits the $\nu_{3}$ plaquette inside the allowed region, in other words giving correct predictions for the MNS mixing elements $U_{\mu 3}$ and $U_{e 3}$. This is because these elements depend only on the vector $\mathbf{v}_{3}=\mathbf{r}\left(m_{\nu_{3}}\right)$ which, as indicated in Figures 6 and 7, is already near the asymptotic value. Hence, the fact the the calculation agrees with data for $U_{\mu 3}$ and $U_{e 3}$ suggests that the rotational fixed point at $\mu=0$ is correctly predicted, although the rotational curve itself near this fixed point is not, by the 1-loop approximation.

In contrast, the state vector $\mathbf{v}_{2}$ of the second heaviest neutrino $\nu_{2}$ represents the tangent vector to the trajectory near the low energy fixed point and cannot therefore be expected to be accurately predicted by the 1-loop calculation of [9]. Indeed, the value predicted by [9] for the mixing element $U_{e 2}$ which depends on $\mathbf{v}_{2}$ fell outside the limits set by the solar neutrino experiments. In our present analysis, the information on $\mathbf{v}_{2}$ extracted from the experimental limits on $U_{e 2}$ can be presented as a wedge-shaped region in Figure 5 in which the tangent to the trajectory at the low energy fixed point is supposed to lie, which region is estimated with a bound $\left|U_{e 2}\right|^{2} \sim 0.33 \pm 0.1$ favoured by present experiments [13, 17]. As can be seen in the figure, the trajectory predicted by the DSM 1-loop calculation does not satisfy this criterion. Again, as in previous cases, there are in fact four solutions to this allowed region, among which we have chosen to display the one which is nearest to accommodating the DSM 1-loop trajectory. However, this is not surprising since it is already expected that the 1-loop trajectory will be unreliable below the $\mu$ mass scale. In that case, it may be interesting turning the argument around to use the information at low scale, scanty though it is at present, to constrain the exact trajectory if such really exists. One sees then that just by deforming somewhat the 1-loop curve, one would be able to remove both the previously noted discrepancies in the $e$ mass and in the mixing element $U_{e 2}$, as indicated in Figures 5, 6 and 7 .

In summary, we conclude that the existing data on fermion mass and mixing when appropriately interpreted do support the hypothesis of a mass matrix rotating with changing scales, and that the rotation trajectory indicated bears a close resemblance to that predicted earlier by the DSM scheme.

We thank Carmen Garcia Garcia for kindly helping us with the best fit to the data presented in Figure 3, and Bill Scott for advising us on the neutrino oscillation data. 


\section{References}

[1] N. Cabibbo, Phys. Rev. Lett. 10, 531 (1963); M. Kobayashi and T. Maskawa, Prog. Teor. Phys. 49, 652 (1973).

[2] See e.g. Chan Hong-Mo hep-th/0007016, Int. J. Mod. Phys, A16, 163, (2001), and/or Chan Hong-Mo and Tsou Sheung Tsun, hep-ph/0008312 (2000), Proc. 8th Asia-Pacific Phys. Conf. APPC 2000, ed. Yeong-Der Yao et al. (World Scientific, Singapore 2001) p. 447.

[3] Z. Maki, M. Nakagawa and S. Sakata, Progr. Theor. Phys. 28, 870 (1962).

[4] C. Jarlskog, in CP Violation, ed. C. Jarlskog, World Scientific, Singapore, 1989.

[5] Steven Weinberg, Phys. Rev. D7, 2887, (1973).

[6] H. Arason, D.J. Castaño, B. Kesthelyi, S. Mikaelian, E.J. Piard, P. Ramond, and B.D. Wright, Phys. Rev. D46, 3945, (1992).

[7] Chan Hong-Mo and Tsou Sheung Tsun, Phys. Rev. D57, 2507, (1998).

[8] Review of Particle Physics, D.E. Groom et al., Eur. Phys. Journ. C15, $1,(2000)$.

[9] José Bordes, Chan Hong-Mo and Tsou Sheung Tsun, hep-ph/9901440, Eur. Phys. J. C 10, 63, (1999).

[10] José Bordes, Chan Hong-Mo and Tsou Sheung Tsun, hep-ph/0104036 (2001).

[11] R. M. Barnett et. al, Phys. Rev. D54, 1 (1996).

[12] José Bordes, Chan Hong-Mo, Jacqueline Faridani, Jakov Pfaudler, and Tsou Sheung Tsun, Phys. Rev. D58, 013004, (1998), hep-ph/9712276.

[13] Superkamiokande data, see e.g. talk by T. Toshito at ICHEP'00, Osaka (2000).

[14] Soudan II data, see e.g. talk by G. Pearce, at ICHEP'00, Osaka (2000).

[15] CHOOZ collaboration, M. Apollonio et al., Phys. Lett. B466, 415, (1999), hep-ex/9907037. 
[16] S.H. Ahm et al., Phys. Lett. B511, 178, (2001), hep-ex/0103001.

[17] Q.R. Ahmad et al. Phys. Rev. Lett. 87, 071307, (2001), nucl-ex/0106015.

[18] G.L. Fogli et al., hep-ph/0106247, Phys. Rev. D64, 0093007 (2001). 